\title{
Optimal design of paired tapered roller bearings under centred radial and axial static loads
}

\author{
EUGENIO DRAGONI ${ }^{\mathrm{a}}$ \\ Dipartimento di Scienze e Metodi dell'Ingegneria, Università degli Studi di Modena e Reggio Emilia, Via Amendola 2, \\ 42122 Reggio Emilia, Italy
}

Received 24 September 2014, Accepted 17 November 2014

\begin{abstract}
Based on the relationships from the ISO 76 standard, this paper optimizes the internal dimensions of tapered roller bearings for maximum static load capacity. A bearing system formed by two identical bearings is assumed, subjected to whatever combination of centred radial and axial forces. It is shown that the static capacity increases linearly with the roller infill, with the ratio of roller length to roller diameter and with the square of the pitch diameter of the roller set. Further, given the ratio of axial to radial force, an optimal contact angle exists which maximizes the static capacity of the bearing pair, regardless of the actual bearing size and ratio of roller diameter to pitch diameter. The optimization procedure can either be used to design custom-made bearings or to pick from manufacturers' catalogues the bearing with the best contact angle for any assigned loading condition.
\end{abstract}

Key words: Radial rolling bearings / tapered rollers / static loading / optimization / design

\section{Introduction}

Ordinarily, rolling bearings are not designed and built in-house but are chosen by the designer from the catalogue of specialized manufactures. The high degree of specialization has fostered the development of standardized high-quality products, readily available off-the-shelf in a wide range of shape and dimensions at affordable prices. Under particular design circumstances, like very large bearings or tight mounting spaces, the need can arise for non-standard bearings which the regular market can satisfy only at a considerable cost of time and money. In such instances, rolling bearings of simple geometry (as with cylindrical or tapered rollers) can be manufactured by the end user itself to meet the specific requirements at a fraction of the costs and delivery time requested by the specialized suppliers.

When tackling the construction of custom bearings the designer has the control of all the variables and the design is conveniently conducted according to optimization methods. Unlike conventional machine elements, for which a wealth of optimization criteria have been developed since long $[1,2]$, the category of rolling bearings has received so far relatively little attention. May be due to the aforementioned passive design approach (selection from a catalogue) towards these components, until the

\footnotetext{
a Corresponding author:

eugenio.dragoni@unimore.it
}

turn of the century the technical literature has been limited to optimal bearing selectors [3] and simulators of bearing kinematics [4]. Papers dealing with the optimization of bearing features have appeared only lately, aimed at maximizing one or several performance properties of ball bearings [5-11], cylindrical roller bearings $[12,13]$ and tapered roller bearings [14-19].

As for ball bearings, Choi and Yoon [5] optimized an automotive wheel-bearing unit with double-row, angularcontact architecture using a genetic algorithm. They showed that the system life can be improved over the standard design without any constraint violations. Kalita et al. [6] adopted a multi-objective optimisation approach for the design of ball bearings with enhanced dynamic capacity, static capacity and lubricant film thickness. The work uses both deterministic methods (penalty functions) and stochastic algorithms (simulated annealing and genetic search). The genetic approach was further explored by Chakraborty et al. [7] for ball bearings and its merits were compared to conventional techniques. Also based on genetic algorithms, a non-linear optimization procedure was developed by Rao and Tiwari [8] for the design of ball bearings with maximum fatigue life under kinematic constraints. The optimized bearing yielded better fatigue life as compared to standard catalogues. This study was further evolved [9] to achieve multi-objective optimization of the bearing in terms of static load capacity, fatigue life and elastohydrodynamic film thickness under inner and 


\section{Nomenclature}

\begin{tabular}{|ll|}
\hline$b$ & Width of outer ring of bearing \\
$C_{0}$ & Static radial load rating of the bearing \\
$d$ & Roller diameter (measured at midlength of roller $)$ \\
$d_{\mathrm{i}}$ & Inside diameter of bearing \\
$d_{\mathrm{o}}$ & Outside diameter of bearing \\
$d_{\delta}^{*}$ & Optimum value of $d$ for $\delta \leq \delta_{\mathrm{lim}}$ \\
$D$ & Pitch diameter of the roller set \\
$D_{\delta}^{*}$ & Optimum value of $D$ for $\delta \leq \delta_{\text {lim }}$ \\
$F_{\mathrm{a}}$ & Axial load acting on the most loaded bearing of the pair \\
$F_{\mathrm{r}}$ & External radial load applied to the bearing pair \\
$F_{\mathrm{rs}}$ & Radial load acting on the single bearing of the pair $\left(=0.5 F_{\mathrm{r}}\right)$ \\
$k$ & Ratio of radial to axial external loads $\left(=K_{\mathrm{a}} / F_{\mathrm{r}}\right)$ \\
$K_{\mathrm{a}}$ & External axial load applied to the bearing pair \\
$L$ & Roller length \\
$L_{\delta}^{*}$ & Optimum value of $L$ for $\delta \leq \delta_{\mathrm{lim}}$ \\
$P_{0}$ & Static equivalent radial load acting on the most loaded bearing of the pair \\
$S_{0}$ & Static safety factor of the bearing pair \\
$s_{0}$ & Intrinsic static safety factor of the bearing pair \\
$s_{0}^{*}$ & Global optimum of $s_{0}$ \\
$s_{0 \delta}^{*}$ & Optimum value of $s_{0}^{*}$ for $\delta \leq \delta_{\text {lim }}$ \\
$X_{0}, Y, Y$ & Load coefficients of the bearing \\
$Z$ & Number of rollers \\
$Z_{\delta}^{*}$ & Optimum value of $Z$ for $\delta \leq \delta_{\text {lim }}$ \\
$\alpha$ & Contact angle of bearing \\
$\alpha^{*}$ & Global optimum of $\alpha$ \\
$\alpha_{\delta}^{*}$ & Optimum value of $\alpha$ for $\delta \leq \delta_{\text {lim }}$ \\
$\delta$ & Pitch ratio of bearing $(=d / D)$ \\
$\delta^{*}$ & Global optimum of $\delta$ \\
$\zeta$ & Filling ratio of the roller set $(=Z d / \pi D)$ \\
$\lambda$ & Aspect ratio of the rollers $(=L / D)$ \\
$\xi$ & Auxiliary variable $(=\delta$ cos $\alpha)$ \\
$\phi$ & Component of the static safety factor $\left(=\zeta \lambda D^{2} / F_{\mathrm{r}}\right)$ \\
$\phi_{\delta}^{*}$ & Optimum value of $\phi$ for $\delta \leq \delta_{\text {lim }}$ \\
&
\end{tabular}

outer geometric constraints. Angular-contact ball bearings were optimized by Savsani et al. [10] for maximum static and dynamic loading ratings and minimum film thickness using a modified particle swarm optimization technique. Both single and multi-objective optimization tasks were considered, claiming advantages with respect to former, mainly genetic, numerical procedures. Wei and Chengzu [11] improved on the computational side of the problem introducing non-dominated sorting genetic algorithms to optimize high-speed, angular-contact ball bearings for fatigue life and frictional power losses.

The optimization of cylindrical roller bearings was first tackled by Kumar et al. [12] for maximum dynamic capacity using real-coded genetic algorithms and adopting, as design variables, the diameter of the rollers, the roller pitch diameter, the roller length and the number of rollers. This work was then expanded by adding the roller crowning (non linear profile) among the design parameters [13] and performing a Monte Carlo sensitivity analysis to investigate changes in the fatigue life of the bearing. The results showed that the multiplier of the logarithmic profile deviation parameter has more effect on the fatigue life as compared with other geometric parameters.
A pioneering instance of tapered roller bearing optimization can be traced back to the paper by Parker et al. [14], in which the performance of several large-bore (about $120 \mathrm{~mm}$ ), tapered roller bearings was simulated and tested at shaft speeds up to $20000 \mathrm{rpm}$ under combined thrust and radial load. The computer-optimized bearing design proved superior to equal-sized standard bearings tested for comparison. Chaturbhuj et al. [15] optimized tapered roller bearings using genetic algorithms and demonstrated that the fatigue life of the bearing improved marginally compared with respect to standard bearings. However, some authors $[12,13]$ have pointed out that some optimization constraints introduced in this paper were unrealistic. A method for optimizing the geometry of tapered roller bearings at high speeds was developed by Walker [16] with the main aim of determining the cup and cone angles which minimize the contact stresses under a specific ratio of axial to radial load. Walker found that at low speeds the optimum cup angle is 40 degrees, whereas the optimum value decreases to 10 degrees for the highest speeds. Wang et al. [17] presented a mathematical model for optimizing the design of four-column bearings with tapered rollers subject to several geometric 


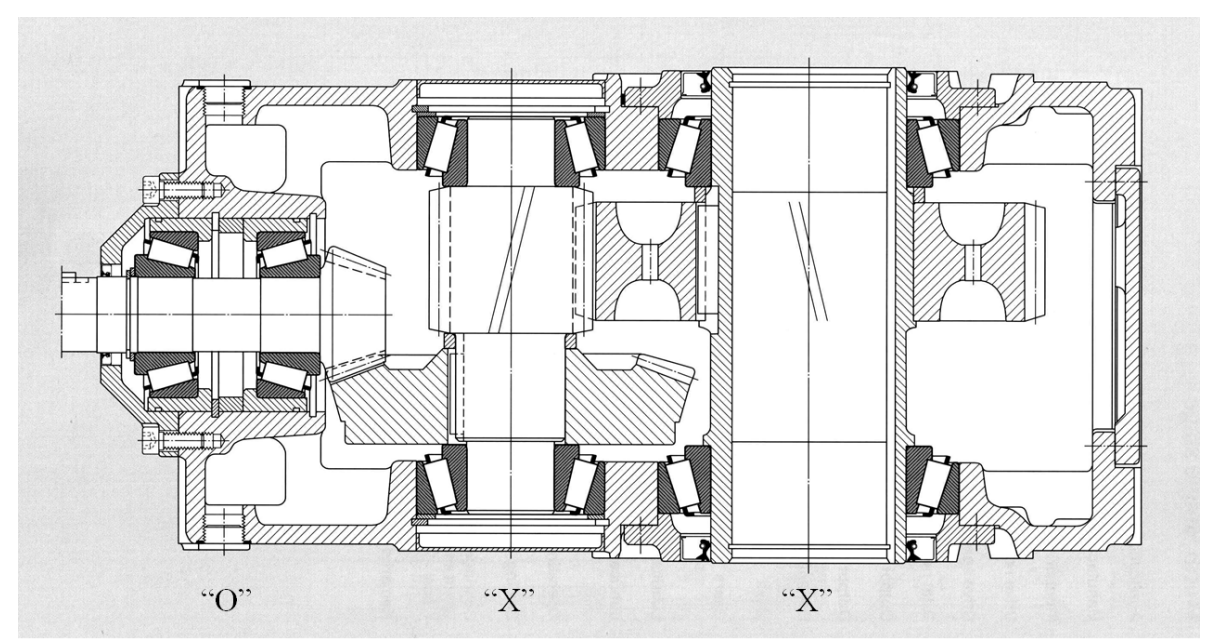

Fig. 1. Longitudinal section of a two-stage bevel/spur gearbox with "O" and "X" arranged tapered roller bearings susceptible of customization and/or optimization.

constraints. By acting on a rearrangement of the classical variables (roller diameter and length, pitch diameter, number of rollers and cup angle), they improved the dynamic load rating by $22 \%$ and the life expectancy by $85 \%$ over a commercial competitor of like dimensions. Though interesting for the potential of improvements it shows, Wang et al.'s paper [17] does not provide details on the optimization method behind the model and little can be taken away from the published results apart from the specific example presented. Two comprehensive contributions to the optimal design of tapered roller bearings have recently been published by Tiwari et al. $[18,19]$. These two papers contain also excellent reviews of the technical literature on rolling bearings and the various optimization methods applied so far to bearing design.

In general, the above papers are focused on the methodological approach to the optimization problem and pay much less attention to the engineering value of the optimization results. If most of the numerical algorithms referenced above can be beneficial to the specialist's work, they are of little use for the general-purpose mechanical designer confronted with the task of designing simple custom bearings. As outcome of an applied research for a small Italian manufacturer of planetary gear drives, the present author has recently published an optimization procedure for radial cylindrical roller bearings [20], which overcomes these limitations. Relying on easy step-by-step calculations and with no need for specific optimization backgrounds, that procedure gives the macro-geometry of the bearing (roller diameter, roller length, pitch diameter of roller set, number of rollers) which maximizes the static and the dynamic load ratings under realistic size constraints.

In the wake of that fruitful effort, this paper tackles the engineering optimization of tapered roller bearings encountered in many industrial applications (see Fig. 1 for a typical instance). The optimization involves the static load capacity of bearing pairs, mounted according to either "O" or "X" arrangements, subjected to a combination of radial and axial forces. Each bearing is defined by the number, diameter and length of the rollers, by the contact angle (cup angle) and by the pitch diameter of the roller set. Following the equations provided by the standard ISO 76 [21], the safety factor of the bearing system is expressed in terms of three parameters: the ratio between roller diameter and pitch diameter, the cup angle and the ratio of axial to radial force. This simple expression gives engineering insight into the problem and shows that for each given load ratio an optimal contact angle exists which maximizes the static load capacity of the bearing, regardless of it actual size and proportions. Tables of optimal contact angles are provided for quick reference.

The optimization method presented here gives its best results for custom-made bearings, for which the design parameters can be varied with the greatest freedom. However, the results disclosed are useful also for identifying the best commercial bearings that can be selected from the manufacturers' catalogues to fit a particular application.

\section{Problem statement}

Figure 2 shows the baseline configuration of the bearing system examined in this paper. The two bearings $B_{1}$ and $B_{2}$ are assumed to be equal and subjected to the radial force $F_{\mathrm{r}}$, which is applied at the centre point of the pair. In addition to $F_{\mathrm{r}}$, an axial thrust $K_{\mathrm{a}}$, also acts on the system through the centre shaft.

Geometrically, each bearing is defined by the following parameters: roller length, $L$; mean roller diameter, $d$ (measured at midlength of $L$ ); number of rollers, $Z$; pitch diameter of the roller set, $D$; contact angle, $\alpha$. The ISO 76 standard [21] specifies that this angle should measure the slope of the raceway without retaining ribs, which is normally the outer one (cup) as shown in Figure 2. Should the retaining rib be provided by the cup, the contact angle $\alpha$ should refer to the inner raceway (cone). 


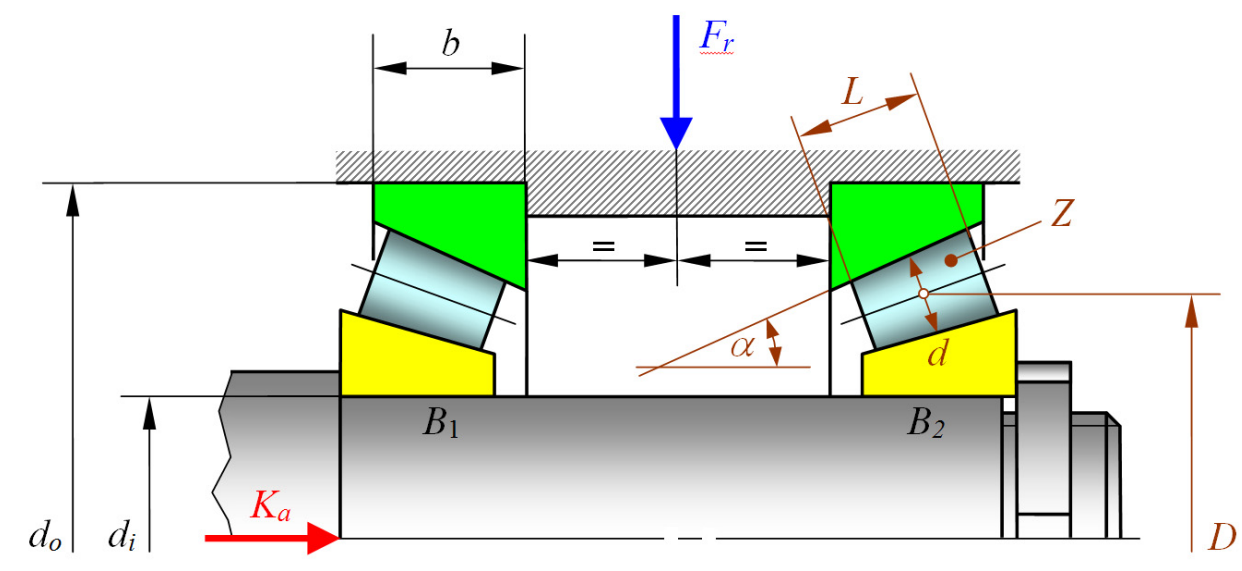

Fig. 2. Reference configuration of the bearing system with applied loads $\left(F_{\mathrm{r}}\right.$ and $\left.K_{\mathrm{a}}\right)$ and characteristic dimensions of the bearings.

With reference to Figure 2, the paper seeks the set of bearing parameters $\{d, D, L, Z, \alpha\}$ which maximizes the static load capacity of the system for given forces $K_{\mathrm{a}}$ and $F_{\mathrm{r}}$. The search for the optimum will be based on the equations provided by the ISO 76 standard [21]. The effect of the flanges is disregarded because the roller-flange contact, though relevant for the wear damage of the bearing, especially for high-speed applications, pays no role under static loading. Although obtained explicitly for the particular bearing combination depicted in Figure 2 (O arrangement with bearings removed from each other), the optimal solution presented will be applicable also to other combinations such as those shown in Figure $3(\mathrm{O}$ and $\mathrm{X}$ arrangements with removed or paired bearings).

\section{Theory}

\subsection{Static radial load rating}

Subject to the conditions clarified below, the standard ISO 76 [21] gives the following field-tested expression for the static radial load rating, $C_{0}(\mathrm{~N})$, of tapered roller bearings

$$
C_{0}=44\left(1-\frac{d}{D} \cos \alpha\right) Z L d \cos \alpha
$$

Equation (1), in which the lengths are expressed in mm, holds true if the bearing is built and installed under the following assumptions: (a) use of bearing steels with hardness HRC $\geq 58$; (b) manufacture according to regular tolerances $[22,23]$ to enhance pressure uniformity over the roller length; (c) accurate guide of rollers with rounded ends to avoid pressure spikes at the edges [24]; (d) mounting onto stiff shafts and within rigid housings; (e) working temperature not higher than $150^{\circ} \mathrm{C}$; (f) an angular load zone of $180^{\circ}$ (i.e. the circumferential extent of the set of rollers that are in contact with both inner and outer raceways). Significant deviations from these reference conditions can be accommodated either by applying correction factors available in the literature [25] or by resorting to second-order methods $[25,26]$ and specialized numerical tools [27] available to bearing manufacturers. The extent of the load zone $f$ is particularly sensitive to the end play (i.e. axial clearance) with which the bearing pair is mounted and to axial displacements produced by the load (see further comments in Sect. 3.2).

Table 1 compares the static load ratings predicted by Equation (1) with the actual load ratings of a selection of tapered roller bearings retrieved from the catalogue of a leading manufacturer (INA). The internal geometry of the bearings in Table 1 (properties from $\alpha$ to $Z$ ) were calculated starting from the catalogue properties (from $d_{i}$ to $\left.Y_{0}\right)$ using the method described in the Appendix.

By defining the filling ratio of the bearing, $\zeta$, the aspect ratio of the rollers, $\lambda$, and the pitch ratio, $\delta$, as follows

$$
\begin{aligned}
\zeta & =\frac{Z d}{\pi D} \\
\lambda & =\frac{L}{d} \\
\delta & =\frac{d}{D}
\end{aligned}
$$

Equation (1) becomes

$$
C_{0}=44 \pi \zeta \lambda D^{2}(1-\delta \cos \alpha) \delta \cos \alpha
$$

Although the theoretical limits for the positive parameters $\zeta, \lambda$ and $\delta$ are $\zeta \leq 1, \lambda \geq 0$ and $\delta<1$, in practice the following ranges are generally observed: $0.5 \leq \zeta \leq 1$, $0.5 \leq \lambda \leq 2$ and $\delta \leq 0.2$.

\subsection{Static equivalent radial load}

Let $F_{\mathrm{rs}}=0.5 F_{\mathrm{r}}$ be the radial load on the single bearing of the system in Figure 2 (remember that $F_{\mathrm{r}}$ is assumed 

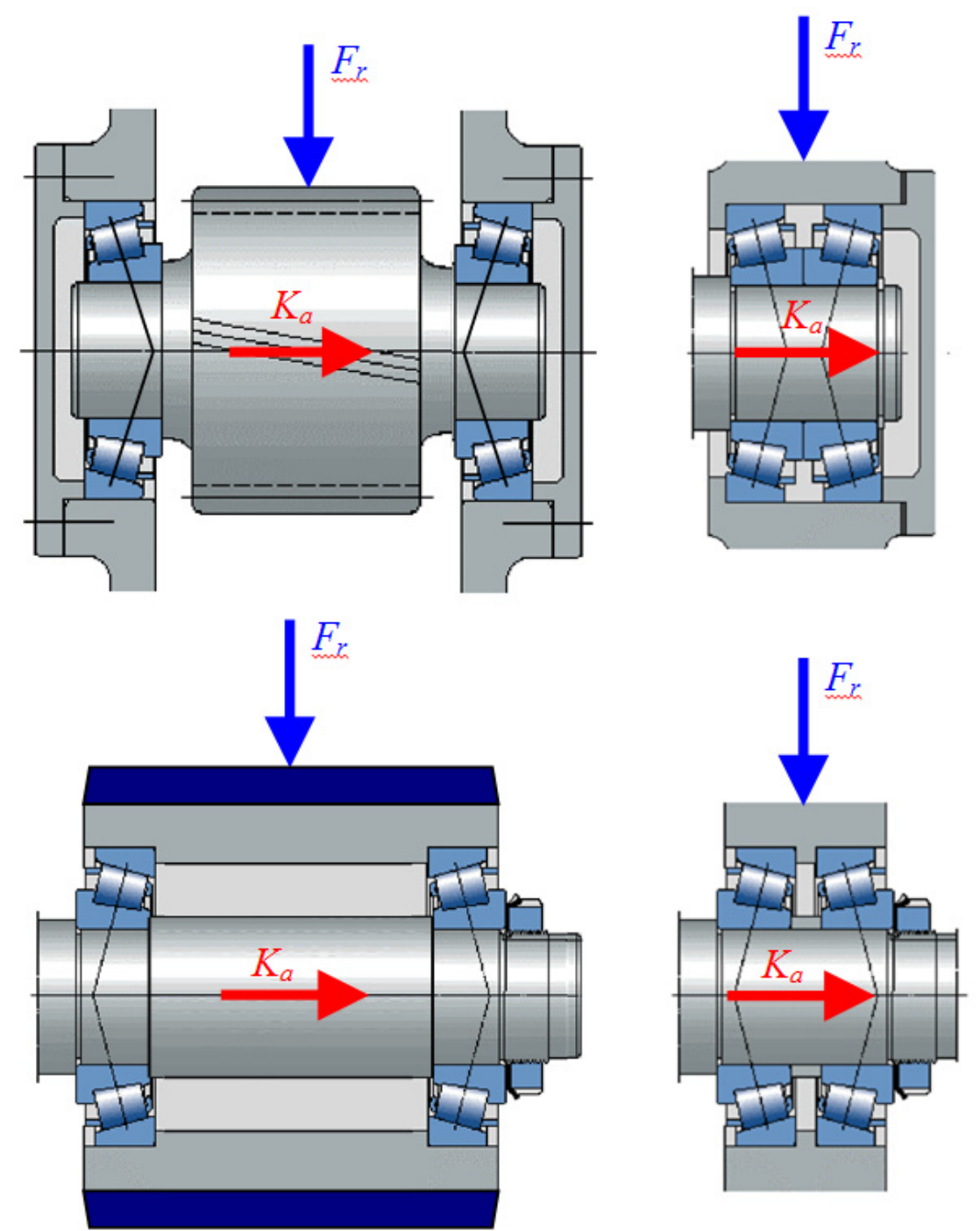

Fig. 3. Examples of bearing combinations to which the present theory is applicable: X-arrangement (top); O-arrangement (bottom); not paired bearings (left); paired bearings (right).

Table 1. Comparison of predicted and catalogue static load ratings for a selection of commercial tapered roller bearings (INA [31]).

\begin{tabular}{cccccccccccc}
\hline \multicolumn{1}{c}{ Properties from INA catalogue } & \multicolumn{4}{c}{ Derived properties (see Appendix) } & \multicolumn{2}{c}{$C_{0}(\mathrm{kN})$} \\
\hline Bearing & $\begin{array}{c}d_{i} \\
(\mathrm{~mm})\end{array}$ & $\begin{array}{c}d_{o} \\
(\mathrm{~mm})\end{array}$ & $\begin{array}{c}b \\
(\mathrm{~mm})\end{array}$ & $\begin{array}{c}Y_{0} \\
(-)\end{array}$ & $\begin{array}{c}\alpha \\
\left({ }^{\circ}\right)\end{array}$ & $\begin{array}{c}d \\
(\mathrm{~mm})\end{array}$ & $\begin{array}{c}D \\
(\mathrm{~mm})\end{array}$ & $\begin{array}{c}L \\
(\mathrm{~mm})\end{array}$ & $\begin{array}{c}Z \\
(-)\end{array}$ & INA & Eq. (1) \\
\hline 30210-A & 50 & 90 & 17 & 0.79 & 15.6 & 10 & 70 & 14.1 & 20 & 96 & 103 \\
30220-A & 100 & 180 & 29 & 0.79 & 15.6 & 20 & 140 & 24.1 & 20 & 325 & 352 \\
30230-A & 150 & 270 & 38 & 0.76 & 16.1 & 30 & 210 & 31.6 & 20 & 630 & 692 \\
30310-A & 50 & 110 & 23 & 0.96 & 12.9 & 15 & 80 & 18.9 & 15 & 148 & 149 \\
30320-A & 100 & 215 & 39 & 0.96 & 12.9 & 28.75 & 157.5 & 32 & 16 & 500 & 519 \\
30330-A & 150 & 320 & 55 & 0.96 & 12.9 & 42.5 & 235 & 45.1 & 16 & 1030 & 1084 \\
31310-A & 50 & 110 & 19 & 0.4 & 28.8 & 15 & 80 & 17.3 & 15 & 125 & 126 \\
31320-X & 100 & 215 & 35 & 0.4 & 28.8 & 28.75 & 157.5 & 32 & 16 & 480 & 476 \\
31330-X & 150 & 320 & 50 & 0.4 & 28.8 & 42.5 & 235 & 45.7 & 16 & 1040 & 1007 \\
T7FC050 & 50 & 105 & 22 & 0.38 & 30.1 & 13.75 & 77.5 & 20.3 & 16 & 135 & 144 \\
T7FC070 & 70 & 140 & 27 & 0.38 & 30.1 & 17.5 & 105 & 25 & 17 & 237 & 242 \\
T7FC095 & 95 & 180 & 33 & 0.38 & 30.1 & 21.25 & 137.5 & 30.5 & 19 & 400 & 406 \\
\hline
\end{tabular}


centred between $B_{1}$ and $\left.B_{2}\right)$ and $F_{\mathrm{a}}$ the actual axial load on the most loaded of the two bearings (bearing $B_{1}$ in Fig. 2). Following ISO 76 [21], the static equivalent radial load acting on the most loaded roller bearing of the pair is

$$
\begin{aligned}
P_{0} & =\operatorname{MAX}\left\{F_{\mathrm{rs}} ; X_{0} F_{\mathrm{rs}}+Y_{0} F_{\mathrm{a}}\right\} \\
& =\operatorname{MAX}\left\{0.5 F_{\mathrm{r}} ; 0.5 X_{0} F_{\mathrm{r}}+Y_{0} F_{\mathrm{a}}\right\}
\end{aligned}
$$

where

$$
X_{0}=0.5 Y_{0}=0.22 \frac{\cos \alpha}{\sin \alpha}
$$

Equation (6) does not account for narrow load zones (see Sect. 3.1) and uses a lower limit corresponding to a load zone of 180 degrees $\left(P_{0}=F_{\mathrm{rs}}\right)$. Another implicit assumption behind Equation (6) is the absence of end moments acting on the shaft at the sections coupled with the bearings, which is consistent with the assumption of rigid shaft. For a flexible shaft, the radial loads on the two bearings would not necessarily be the same due to interdependence between radial displacements, tilt rotations and unequal load zones. Handling of these situations would necessarily call for the use of in-house tools developed by bearing manufacturers (e.g. [27]).

For a rigid shaft under the centre radial loading in Figure 2 and assuming neither end play nor axial preload on the system, the maximum axial force $F_{\mathrm{a}}$ is given by the following universally accepted equation [28]

$$
F_{\mathrm{a}}=K_{\mathrm{a}}+0.5\left(\frac{F_{\mathrm{rs}}}{Y}\right)=K_{\mathrm{a}}+0.25 \frac{F_{\mathrm{r}}}{Y}
$$

with [28]

$$
Y=0.4 \frac{\cos \alpha}{\sin \alpha}
$$

Using professional tools, it can be shown that an axial assembly preload equal to $F_{\mathrm{a}} / F_{\mathrm{rs}} \approx 1.55$ would slightly improve the load carrying capacity of the bearing system with respect to the assumption of no end play. By contrast, a positive end play (clearance) or the axial displacement induced by the load itself would dramatically decrease the load zone of the secondary bearing (i.e. the bearing which does not support directly the external axial load, $B_{2}$ in Fig. 2), so that it could become the element of the pair which experiences the highest contact stresses. For this reason the end play should always be strictly controlled and positive values should be avoided whenever possible.

Using (7)-(9) and introducing the load ratio, $k$, as

$$
k=\frac{K_{\mathrm{a}}}{F_{\mathrm{r}}}
$$

Equation (6) becomes

$$
P_{0}=F_{\mathrm{r}} \operatorname{MAX}\left\{0.5 ; 0.25+0.22\left(\frac{\cos \alpha}{\sin \alpha} k+0.625\right)\right\}
$$

\subsection{Static safety factor}

ISO 76 [21] defines the static safety factor, $S_{0}$, as

$$
S_{0}=\frac{C_{0}}{P_{0}}
$$

By means of (5) and (11), Equation (12) gives

$$
S_{0}=\frac{\zeta \lambda D^{2}}{F_{\mathrm{r}}} \frac{44 \pi(1-\delta \cos \alpha) \delta \cos \alpha}{\operatorname{MAX}\left\{0.5 ; 0.25+0.22\left(\frac{\cos \alpha}{\sin \alpha} k+0.625\right)\right\}}
$$

which can be written as:

$$
S_{0}=\phi s_{0}
$$

with

$$
\phi=\frac{\zeta \lambda D^{2}}{F_{\mathrm{r}}}
$$

and

$$
s_{0}=\frac{44 \pi(1-\delta \cos \alpha) \delta \cos \alpha}{\operatorname{MAX}\left\{0.5 ; 0.25+0.22\left(\frac{\cos \alpha}{\sin \alpha} k+0.625\right)\right\}}
$$

\section{Optimization}

\subsection{Free optimization}

Equation (14) shows that the static safety factor, $S_{0}$, is proportional to the functions $\phi$ and $s_{0}$. From Equation (15) we see that, for given radial load $F_{\mathrm{r}}$, function $\phi$ increases linearly with the filling ratio, $\zeta$, and the aspect ratio, $\lambda$, and goes up quadratically with the pitch diameter, $D$. Function $\phi$ can be regarded as a control factor through which the safety factor, $S_{0}$, can easily be made large at will by increasing the pitch diameter. Similarly, function $s_{0}$ in Equation (14) can be interpreted as an intrinsic safety factor of the bearing system obtained when the filling ratio, the aspect ratio, the pitch diameter and the radial load assume unit value. From Equation (16) we see that $s_{0}$ depends non-linearly on $\alpha, \delta$ and $k$ as shown by the three-dimensional charts in Figure 4.

Using $s_{0}$ as objective function, the free optimization problem can be stated as follows: Maximize $s_{0}(\bar{X})$, subjected to $k=$ const. $\left(=K_{\mathrm{a}} / F_{\mathrm{r}}\right)$, with $\bar{X}=(\delta, \alpha)$. Maximization of $s_{0}$ implies minimization of the denominator and maximization of the numerator in Equation (16). For any given load ratio $k$, the denominator of Equation (16) achieves the absolute minimum value 0.5 when the following condition is met

$$
0.25+0.22\left(\frac{\cos \alpha}{\sin \alpha} k+0.625\right)=0.5
$$

from which the optimum contact angle, $\alpha^{*}$ is obtained as:

$$
\tan \alpha^{*}=\frac{88}{45} k \approx 1.956 k
$$


E. Dragoni: Mechanics \& Industry 16, 604 (2015)
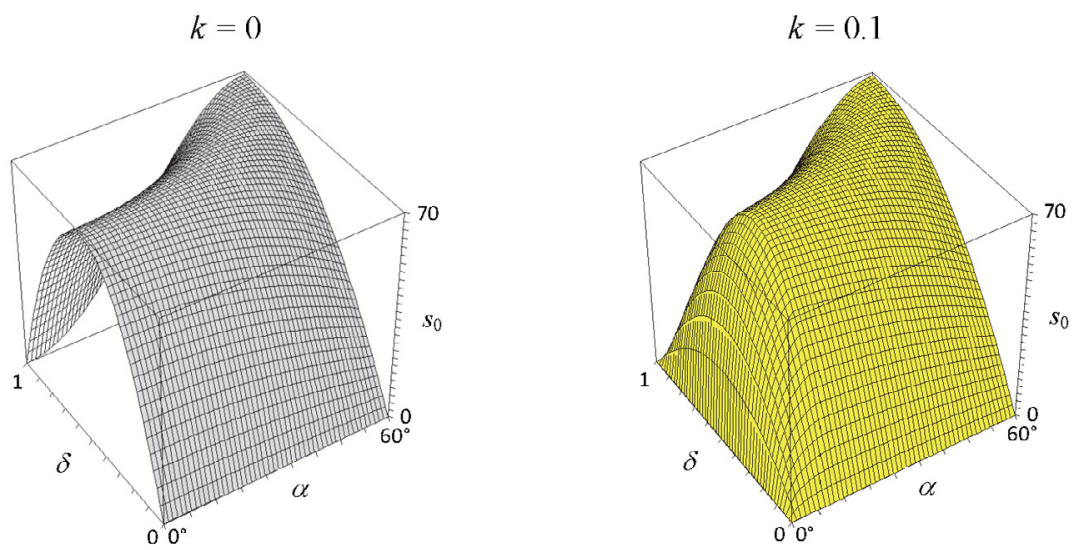

$k=0.2$
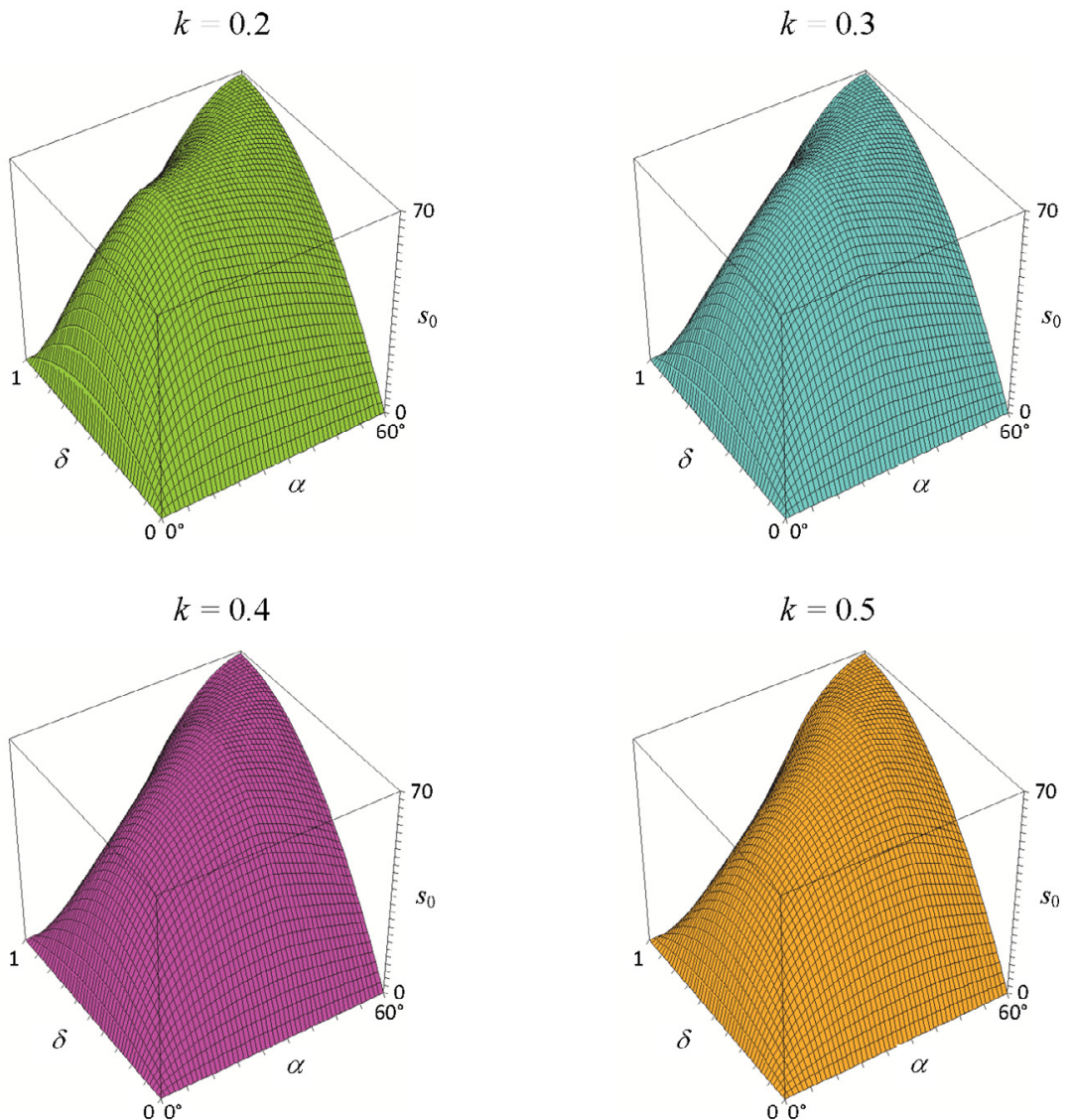

Fig. 4. Charts of the intrinsic safety factor $s_{0}$ for a selection of load ratios $k$.

Letting $\xi=\delta \cos \alpha$, the numerator of Equation (16) is maximum when

$$
\frac{\mathrm{d}}{\mathrm{d} \xi}[(1-\xi) \xi]=0
$$

giving

$$
\xi=\delta \cos \alpha=0.5
$$

Combination of Equations (18) and (20) yields the optimal pitch ratio $\delta^{*}$ as

$$
\delta^{*}=\frac{0.5}{\cos \left\{\arctan \left(\frac{88}{45} k\right)\right\}}
$$

Introducing Equations (18) and (21) into (16) gives the following absolute maximum value, $s_{0}^{*}$, for the intrinsic safety factor

$$
s_{0}^{*}=22 \pi \approx 69.115
$$

The optimal contact angles and pitch ratios given by Equations (18) and (21) are collected in Table 2 for the load ratio $k$ ranging from 0 to 1 . Figure 5 depicts the shape assumed by the optimal bearing for $k=0, k=0.3$ and $k=0.6$. 


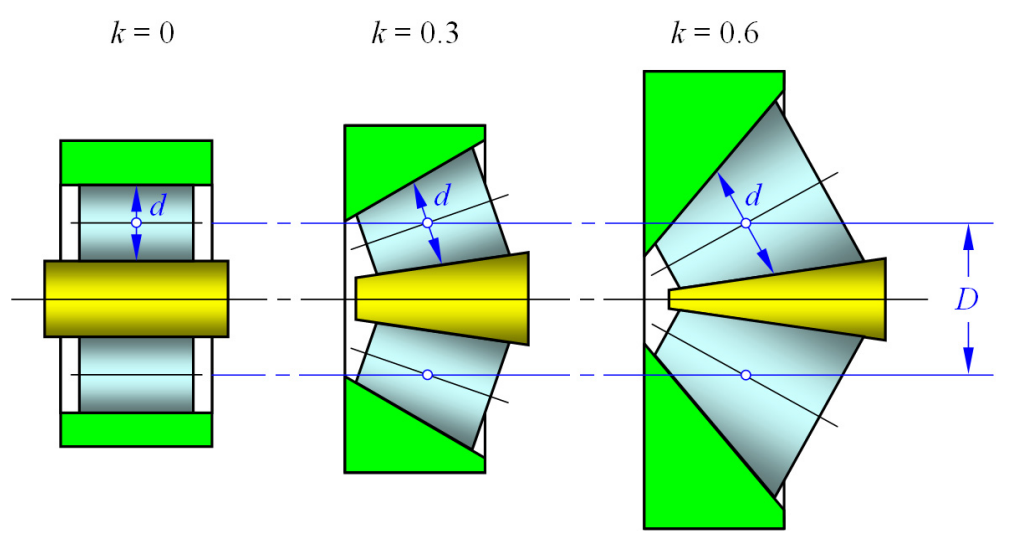

Fig. 5. Optimal bearings for different load ratios $k$ and constant pitch diameter $D$ when no limits are put to the pitch ratio $\delta=d / D$.

Table 2. Absolute optimum values of contact angle $\left(\alpha^{*}\right)$ and pitch ratio $\left(\delta^{*}\right)$ for a range of load ratios $(k)$.

\begin{tabular}{cccc}
\hline$k$ & $\tan \alpha^{*}$ & $\alpha^{*}\left({ }^{\circ}\right)$ & $\delta^{*}$ \\
\hline 0 & 0 & 0 & 0.50 \\
0.1 & 0.196 & 11.1 & 0.51 \\
0.2 & 0.391 & 21.4 & 0.54 \\
0.3 & 0.587 & 30.4 & 0.58 \\
0.4 & 0.782 & 38.0 & 0.64 \\
0.5 & 0.978 & 44.4 & 0.70 \\
0.6 & 1.173 & 49.6 & 0.77 \\
0.7 & 1.389 & 53.8 & 0.85 \\
0.8 & 1.564 & 57.4 & 0.93 \\
0.9 & 1.760 & 60.4 & 1.01 \\
1.0 & 1.956 & 62.9 & 1.10 \\
\hline
\end{tabular}

\subsection{Constrained optimization}

The proportions of the optimal bearings in Figure 5 are too cumbersome, especially for $k>0.3$, to be of practical use. A more fruitful approach is obtained by stating the optimization problem with a constraint on the pitch ratio as follows: Maximize $s_{0}(\bar{X})$, subjected to $\delta \leq \delta_{\lim }$ and $k=$ const. $\left(=K_{\mathrm{a}} / F_{\mathrm{r}}\right)$, with $\bar{X}=(\delta, \alpha)$. This problem is plotted for several $k$ in Figure 6 , with the contour lines of $s_{0}$ drawn as a function of the contact angle, $\alpha$, and the pitch ratio, $\delta$. The centre curve $m n$ in Figure 6 corresponds to the global optimum safety factor $s_{0}=s_{0}^{*}$ given by Equation (22). For any chosen load ratio $k$, the coordinates of point $m$ in Figure 6 provide the global optimal values $\alpha^{*}$ and $\delta^{*}$ listed in Table 2 .

Figure 6 shows that, for reasonable values of the limit pitch ratio (i.e. $\delta_{\lim } \leq 0.5$ ), an optimal contact angle, $\alpha_{\delta}^{*}$, always exists, which depends on the limit pitch ratio itself. Take, for example the chart in Figure 6 for $k=0.4$ and assume $\delta \leq \delta_{\text {lim }}=0.3$ meaning that only the region below line $a b$ is feasible. The greatest value of $s_{0}$ that can be achieved in that region is obtained by moving on line $a b$ and sweeping the contact angle from $a$ to $b$ until the optimum point $M$ is reached. Point $M$ is defined as the tangent point between $a b$ itself and whichever contour line occurs to be touching the line $a b$. The abscissa of point $M$ gives the optimal value $\alpha_{\delta}^{*}$ for the contact angle $\left(\alpha_{\delta}^{*} \approx 35^{\circ}\right.$ in this example). The contour line of $s_{0}$ passing from $M$ gives the corresponding intrinsic safety factor $\left(s_{0 \delta}^{*} \approx 50\right.$ in the example).

This constrained optimization can be performed systematically once and for all using Equation (16) for any combination of load ratios $k$ and pitch ratios $\delta$. For given $k$ and $\delta$, the intrinsic safety factor $s_{0}$ in Equation (16) depends only on $\alpha$ and the optimum value $\alpha_{\delta}^{*}$ is easily found numerically. Optimal values of $\alpha_{\delta}^{*}$ and $s_{0 \delta}^{*}$ obtained in this way are reported in Table 3 for load ratios in the range $0 \leq k \leq 1.0$ and pitch ratios $0.05 \leq \delta \leq 0.25$ (the most likely to occur in practice). Optimal bearings for $\delta_{\lim }=0.2$ are shown in Figure 7 for $k=0\left(\alpha_{\delta}^{*}=0^{\circ}\right)$, $k=0.3\left(\alpha_{\delta}^{*}=30^{\circ}\right)$ and $k=0.6\left(\alpha_{\delta}^{*}=38^{\circ}\right)$. In marked contrast with the bearings in Figure 6, awkward and hardly feasible, the designs in Figure 7 are sleek and technically viable.

The use of Table 3 for the optimal design of tapered roller bearings is easily performed as clarified by the following example. Assume that the bearing system in Figure 2 has to be designed for the loads $F_{\mathrm{r}}=600000 \mathrm{~N}$ and $K_{\mathrm{a}}=180000 \mathrm{~N}$ with a safety factor $S_{0}=1.5$. Calculating from Equation (10) $k=K_{\mathrm{a}} / F_{\mathrm{r}}=600000 / 180000=0.3$ and assuming a limit pitch ratio $\delta_{\lim }=0.15$, Table 3 gives the optimal contact angle $\alpha_{\delta}^{*}=30^{\circ}$ and the optimal intrinsic safety factor $s_{0 \delta}^{*}=31.14$. From Equation (14) the value $\phi_{\delta}^{*}=S_{0} / s_{0 \delta}^{*}=1.5 / 31.14 \approx 0.0482$ is calculated, which, adopting a filling ratio $\zeta=0.8$ (Eq. (2)) and an aspect ratio $\lambda=1.5$ (Eq. (3)) and using (15), gives $D_{\delta}^{*}=\left(\phi_{\delta}^{*} F_{\mathrm{r}} / \zeta \lambda\right)^{0.5}=(0.0482 \times 600000 / 0.8 \times 1.5)^{0.5} \approx$ $155 \mathrm{~mm}$. From Equations (4), (3) and (2), the optimal mean roller diameter, optimal roller length and optimal number of rollers are finally obtained as $d_{\delta}^{*}=\delta_{\lim } D_{\delta}^{*}=$ $0.15 \times 155 \approx 23.3 \mathrm{~mm}, L_{\delta}^{*}=\lambda d_{\delta}^{*}=1.5 \times 23.3 \approx 35 \mathrm{~mm}$, $Z_{\delta}^{*}=\pi \zeta D_{\delta}^{*} / d_{\delta}^{*}=\pi \times 0.8 \times 155 / 23.3 \approx 17$.

\section{Discussion}

\subsection{Review of the results}

Table 1 shows that, despite its simplicity, Equation (1) predicts quite accurately the static load ratings of com- 
E. Dragoni: Mechanics \& Industry 16, 604 (2015)

$k=0$

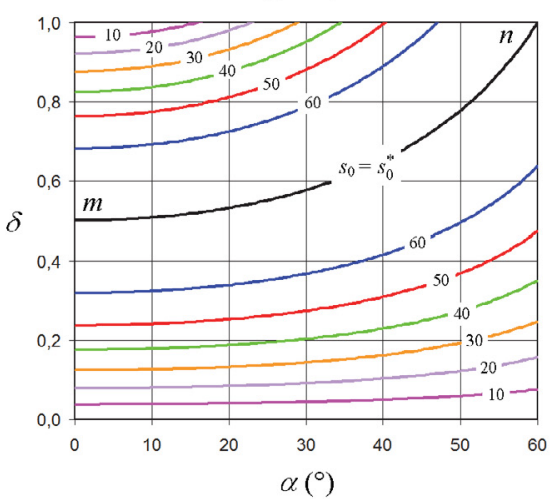

\section{$k=0.2$}

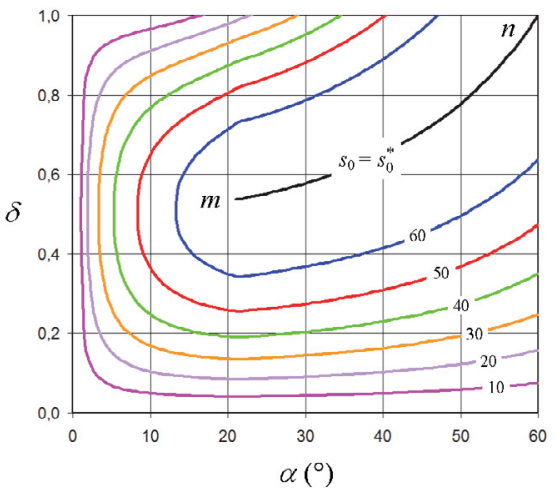

$k=0.4$

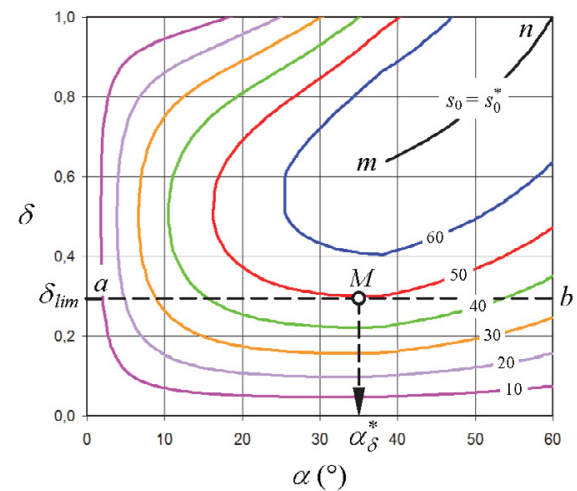

$k=0.1$

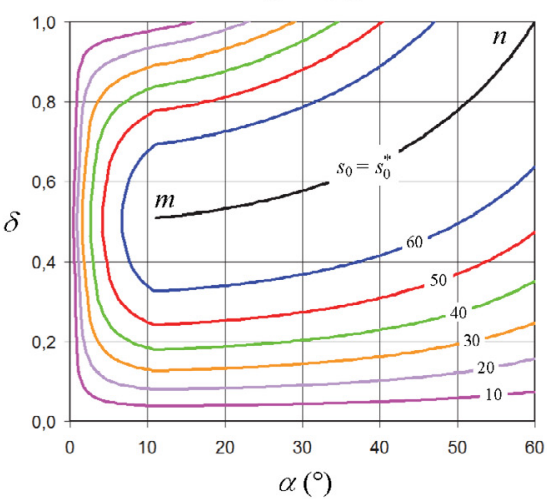

$k=0.3$

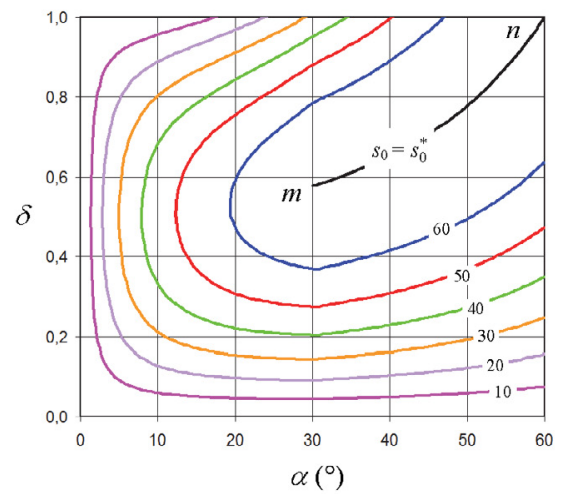

$k=0.5$

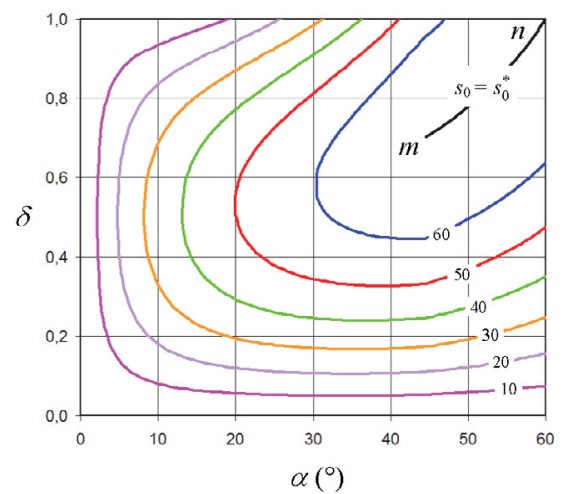

Fig. 6. Contour lines of the intrinsic safety factor $s_{0}$ for a selection of load ratios $k$.

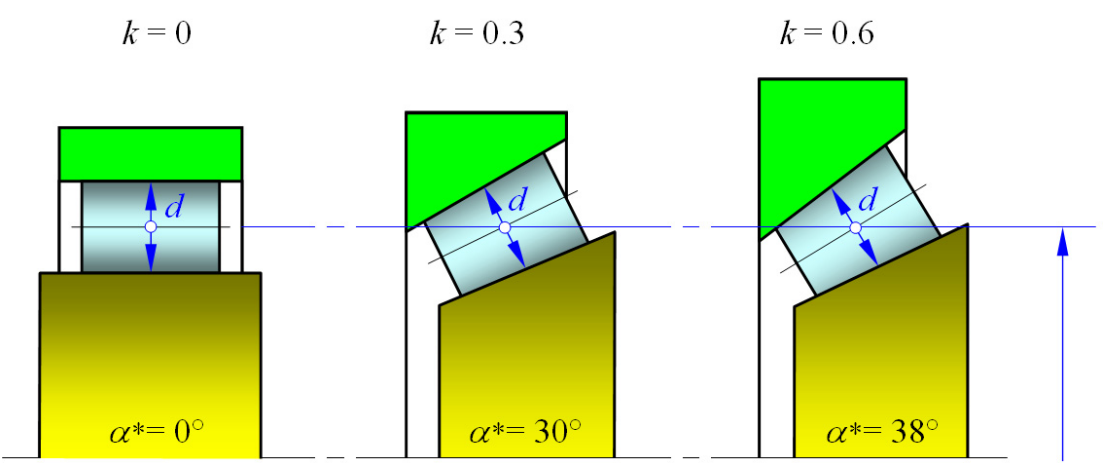

Fig. 7. Optimal bearings for different load ratios $k$ and limit pitch ratio $\delta_{\lim }=0.2$. 
Table 3. Optimum values of contact angle $\left(\alpha_{\delta}^{*}\right)$ and corresponding intrinsic safety factors $\left(s_{0 \delta}^{*}\right)$ for given load ratios $(k)$ and limit pitch ratios $\left(\delta_{\lim }\right)$.

\begin{tabular}{|c|c|c|c|c|c|c|c|c|c|c|}
\hline \multicolumn{11}{|c|}{$\delta_{\lim }$} \\
\hline \multirow[b]{2}{*}{$k$} & \multicolumn{2}{|c|}{0.05} & \multicolumn{2}{|c|}{0.1} & \multicolumn{2}{|c|}{0.15} & \multicolumn{2}{|c|}{0.2} & \multicolumn{2}{|c|}{0.25} \\
\hline & $\alpha_{\delta}^{*}\left({ }^{\circ}\right)$ & $s_{0 \delta}^{*}$ & $\alpha_{\delta}^{*}\left({ }^{\circ}\right)$ & $s_{0 \delta}^{*}$ & $\alpha_{\delta}^{*}\left({ }^{\circ}\right)$ & $s_{0 \delta}^{*}$ & $\alpha_{\delta}^{*}\left({ }^{\circ}\right)$ & $s_{0 \delta}^{*}$ & $\alpha_{\delta}^{*}\left({ }^{\circ}\right)$ & $s_{0 \delta}^{*}$ \\
\hline 0 & 0 & 13.13 & 0 & 24.88 & 0 & 35.25 & 0 & 44.23 & 0 & 51.84 \\
\hline 0.1 & 11.0 & 12.89 & 11.0 & 24.44 & 11 & 34.67 & 11.0 & 43.56 & 11.0 & 51.14 \\
\hline 0.2 & 21.5 & 12.26 & 21.5 & 23.33 & 21.5 & 33.20 & 21.5 & 41.87 & 21.5 & 49.35 \\
\hline 0.3 & 29.5 & 11.41 & 30.0 & 21.79 & 30.0 & 31.14 & 30.0 & 39.45 & 30.0 & 46.73 \\
\hline 0.4 & 32.0 & 10.62 & 32.5 & 20.31 & 33.0 & 29.07 & 34.0 & 36.91 & 35.0 & 43.87 \\
\hline 0.5 & 34.0 & 9.98 & 34.5 & 19.09 & 35.0 & 27.36 & 36.0 & 34.79 & 37.0 & 41.41 \\
\hline 0.6 & 35.5 & 9.43 & 36.0 & 18.06 & 37.0 & 25.90 & 38.0 & 32.98 & 39.0 & 39.31 \\
\hline 0.7 & 37.0 & 8.95 & 37.5 & 17.17 & 38.5 & 24.65 & 39.5 & 31.41 & 40.5 & 37.48 \\
\hline 0.8 & 38.5 & 8.54 & 39.0 & 16.38 & 39.5 & 23.54 & 40.5 & 30.03 & 41.5 & 35.87 \\
\hline 0.9 & 39.5 & 8.17 & 40.0 & 15.68 & 41.0 & 22.55 & 41.5 & 28.80 & 42.5 & 34.43 \\
\hline 1.0 & 40.5 & 7.84 & 41.0 & 15.06 & 42.0 & 21.67 & 42.5 & 27.69 & 43.5 & 33.14 \\
\hline
\end{tabular}

mercial tapered roller bearings. Within the wide range of dimensions and contact angles considered, the maximum error in Table 1 (see Appendix) is less than 10 per cent (bearing 3023-A) while the average absolute error is just above 4 per cent. Errors of the same order of magnitude were calculated by Dragoni [20] for one standard cylindrical roller bearing for which the internal dimensions were known exactly, not simply estimated as in Table 1.

For the case of purely radial load $(k=0)$, Table 2 gives a global optimum solution with contact angle $\alpha^{*}=0$ and pitch ratio $\delta^{*}=0.5$, which means cylindrical rollers of diameter, $d$, equal to one half of the pitch diameter, $D$. This result coincides with the global optimal proportions reported by Dragoni [20] for the specific category of radial cylindrical roller bearings. For increasing axial loads $(k>0)$, the global optima for the pitch ratio collected in Table 2 become more and more unlikely for real-life applications (see Fig. 5). The reason why the theoretical optimization tends to these quite odd shapes is perhaps imputable to the fact that the empirical expression (1) was developed to fit the experimental behaviour of bearings with pitch ratios much lower than 0.5 as commonly encountered in practice (for example, the pitch ratios of the twelve commercial bearings in Tab. 1 range from 0.14 to 0.19$)$.

Table 3, developed to take into account realistic geometric constraint on $\delta\left(\delta \leq \delta_{\lim }=0.05 \ldots 0.25\right)$, shows that the optimum contact angle, $\alpha_{\delta}^{*}$, depends both on the load ratio, $k$, and the limit pitch ratio, $\delta_{\text {lim }}$. However, while the effect of the load ratio is strong (with $\alpha_{\delta}^{*}$ increasing monotonically with $k$ ), the dependence on the pitch ratio is weak (on passing from $\delta_{\text {lim }}=0.05$ to $\delta_{\text {lim }}=0.25$, the maximum relative increase of the optimum contact angle is about $10 \%$ and occurs for $k=0.6)$. For $k=0.3$, the optimal contact angle in Table 3 is about $30^{\circ}$, which is the highest contact angle prescribed by the ISO 355 standard [29] and offered by most bearing manufacturers (see Tab. 1). For load ratios greater than 0.3 , the optimal contact angle exceeds $30^{\circ}$ and reaches the optimum value slightly above $40^{\circ}$ for $k=1$. In this range of operation, special supplies [30] or custom constructions are needed to achieve the maximum load capacity.

Walker [16] confirms that for low-speed roller bearings loaded under prevailing axial thrusts, the optimal cone angle is about $40^{\circ}$ as predicted by Table 1 for $k \geq 0.8$. Conversely, for $k=0$, purely radial load, Table 1 predicts correctly $\alpha^{*}=0^{\circ}$ and Equation (21) predicts $\delta^{*}=0.5$, meaning cylindrical rollers with diameter one half of the pitch diameter. This result coincides with the global optimum reported by Dragoni [20] for the specific category of radial cylindrical roller bearings.

Table 3 also shows that the optimal intrinsic safety factor, $s_{0 \delta}^{*}$, rapidly increases with the limit pitch ratio, $\delta_{\lim }$, and decreases with the load ratio, $k$. The increase of $s_{0 \delta}^{*}$ with $\delta_{\text {lim }}$ is a consequence of the marked gradient of the surfaces of $s_{0}$ in Figure 4 (confirmed by the density of the contour lines in Fig. 6) for pitch ratios in the range $0 \leq \delta \leq 0.5$. The decrease of $s_{0 \delta}^{*}$ with $k$ is due to the fact that, given the radial force $F_{\mathrm{r}}$ in Figure 2, an increase of the load ratio $k$ implies a greater total force on the bearing system with respect to the condition of pure radial loading.

With reference to the numerical example at the end of the Section Constrained optimization, it is easily verified that substituting the design data $F_{\mathrm{r}}=600 \mathrm{kN}$ and $k=$ 0.3 , together with the optimal results $\alpha_{\delta}^{*}=30^{\circ}, d_{\delta}^{*}=$ $23 \mathrm{~mm}, L_{\delta}^{*}=34.5 \mathrm{~mm}, Z_{\delta}^{*}=17$ for $F_{\mathrm{r}}, k, \alpha, d, L$ and $Z$, in Equations (11) and (1), gives $P_{0}=300 \mathrm{kN}$ and $C_{0}=458.8 \mathrm{kN}$, respectively. These values imply a safety factor $S_{0}=C_{0} / P_{0}=458.8 / 300 \approx 1.53$, slightly greater than the design value of 1.5 . This small difference is due to roundoff of the variables involved in the calculations, especially as regards to the optimum number of rollers $Z_{\delta}^{*}$ (the exact value 16.72 was rounded to 17 in the example). 
E. Dragoni: Mechanics \& Industry 16, 604 (2015)

Table A.1. Relationships between catalogue data $\left(Y_{0}, d_{i}, d_{o}, b\right)$ and internal bearing properties $(\alpha, d, D, L, Z)$.

\begin{tabular}{cccc}
\hline Internal property & Relationship & Numerical values & Source \\
\hline$\alpha$ & $=\arctan \left(\frac{0.22}{Y_{0}}\right)$ & - & Equation $(7)$ \\
$d$ & $=q_{1}\left(d_{o}-d_{i}\right)$ & $q_{1}=0.25$ & Textbook [32] \\
$D$ & $=q_{2}\left(d_{o}+d_{i}\right)$ & $q_{2}=0.5$ & Assumption \\
$L$ & $=q_{3} \frac{b}{\cos \alpha}$ & $q_{3}=0.8$ & Assumption \\
$Z$ & $=q_{4} \frac{\left(d_{o}+d_{i}\right)}{d}$ & $q_{4}=1.45$ & Textbook [32] \\
\hline
\end{tabular}

\subsection{Limitations of the model and future work}

The present optimization is built on the assumption that the critical bearing of the pair is the one that directly supports the axial load ( $B_{1}$ in Fig. 2$)$. This is the natural consequence of using Equation (6) for calculating the maximum equivalent load on the system. Though this approach is coherent with the design formulae present in the manufacturers' catalogues, it has limitations when the bearing pair is mounted with end play (axial clearance) or undergoes large axial deformations under load. A large axial load induces a large axial displacement which causes a very narrow load zone in the second bearing $\left(B_{2}\right.$ in Fig. 2, not considered here), hence generating risk of large roller-race load and pressure. Under these unfavourable working conditions, the second bearing may thus become the critical element, which is not optimized here. In this case, the full answer to the problem cannot be obtained with the present analytical model and requires numerical tools considering equilibrium and compatibility (deformations) of bearings, shaft and housing as a whole.

In practical terms, the optimal design described in this paper strictly holds true when the bearing system is assembled with a light preload that would compensate the axial displacement induced in the secondary bearing by the externally applied load. This is certainly a limitation, but not a prohibitive one since preloading of tapered roller bearings is a common procedure for the many advantages it brings about such as (a) increase of the stiffness, (b) noise reduction, (c) improved rotational precision, (d) wear compensation, and (e) longer life.

Spurred by the insightful results obtained for the static loading covered in this paper, the next step of the research will focus on the optimization of the bearing system in Figure 2 for maximum dynamic load-carrying capacity. In the light of the comments on the sensitivity of the contact zone emphasized in this section, an effort will be made to incorporate the effects of the bearing deformation into the optimization procedure.

\section{Conclusion}

Using the empirical relationships provided by the ISO 76 standard, the internal dimensions of tapered roller bearings are optimized for maximum static load capacity. The bearing system investigated comprises two identical bearings undergoing whatever combination of radial and axial forces. Assuming that the radial force is applied at equal distance from the bearings of the pair and that neither end play (axial clearance) nor abnormal preloading affect the assembly, the closed-form optimization process leads to the following general results:

- the static load capacity increases linearly with the filling ratio (number of rollers divided by the maximum number which can fill the bearing) and the aspect ratio (ratio of roller length to mean roller diameter) and goes up with the square of the pitch diameter of the roller set;

- given the ratio of axial to radial force, global optima exist for the contact angle and the pitch ratio (ratio of roller diameter to pitch diameter) which maximize the static load capacity;

- the bearing proportions at the global optima are too sturdy (contact angles greater than 60 degrees, pitch ratios equal to or greater than 0.5 ) to be used in practice;

- if the pitch ratio is constrained to stay below reasonable limits $(\leq 0.25)$, an optimal contact angle exists which maximizes the static load capacity, regardless of the actual size and proportions of the bearing;

- the results of the optimization are conveniently summarized by a general table and a few simple equations that can be followed step-by-step to design the optimal bearing that suits any given application;

- the optimization procedure can either be used to design custom-made bearings (thus exploiting the geometrical freedom to the full) or to pick from the manufacturers' catalogues the bearing with the best contact angle for any assigned loading.

\section{Appendix: Internal dimensions of tapered roller bearings}

The internal dimensions of rolling bearings are proprietary data which the manufacturers do not provide in their catalogues. However, starting from the external dimensions of the bearings $\left(d_{i}, d_{o}, b\right.$ in Fig. 2) and the static coefficient, $Y_{0}$, available from the catalogues, the internal properties ( $\alpha, d, D, L, Z$ in Fig. 2) can be calculated within $10-15$ per cent of the true value. Table A.1 shows how the internal properties displayed in Table 1 were obtained step-by-step from the catalogue data using elementary geometrical considerations and characteristic proportions available from technical textbooks [32]. 


\section{References}

[1] A. Seireg, A survey of optimisation of mechanical design, J. Eng. Ind. T ASME 94 (1972) 495-499

[2] A.A. Seireg, J. Rodriguez, Optimizing the shape of mechanical elements and structures, CRC Press, Cleveland, OH, 1997

[3] J. Ahluwalia, S.K. Gupta, V.P. Agrawal, Computer-aided optimum selection of roller bearings, Comput. Aid. Des. 25 (1993) 493-499

[4] H. Aramaki, Rolling bearing analysis program package BRAIN, NSK Technical Journal Motion Control 3 (1997) $15-24$

[5] D.-H. Choi, K.-C. Yoon, A Design Method of an automotive wheel-bearing unit with discrete design variables using genetic Algorithms, J. Tribol. T ASME 123 (2001) 181-187

[6] K. Kalita, R. Tiwari, S.K. Kakoty, Multi-objective optimisation in rolling element bearing system design, In: Proceedings of the International Conference on Optimisation (SIGOPT 2002), Lambrecht, Germany, 2002

[7] I. Chakraborty, V. Kumar, S.B. Nair, R. Tiwari, Rolling element bearing design through genetic algorithms, Eng. Optimiz. 35 (2003) 649-659

[8] B.R. Rao, R. Tiwari, Optimum design of rolling element bearings using genetic algorithms, Mech. Mach. Theory 42 (2007) 233-250

[9] S. Gupta, R. Tiwari, S.B. Nair, Multi-objective design optimisation of rolling bearings using genetic Algorithms, Mech. Mach. Theory 42 (2007) 1418-1443

[10] V. Savsani, R.V. Rao, D.P. Vakharia, Multi-objective design optimization of ball bearings using a modified particle swarm optimization technique, Int. J. Des. Eng. 1 (2008) 412-433

[11] Y. Wei, R. Chengzu, Optimal design of high speed angular contact ball bearing using a multiobjective evolution algorithm, In: Proceedings of the IEEE Int. Conf. on Computing, Control and Industrial Engineering (CCIE 2010), Wuhan, China, 2010

[12] K.S. Kumar, R. Tiwari, R.S. Reddy, Development of an optimum design methodology of cylindrical roller bearings using genetic algorithms, Int. J. Comput. Meth. Eng. Sci. Mech. 9 (2008) 321-341

[13] K.S. Kumar, R. Tiwari, V.V.N. Prasad, An optimum design of crowned cylindrical roller bearings using genetic Algorithms, J. Mech. Des. T ASME 131 (2009) 051011-1051011-14

[14] R.J. Parker, S.I. Pinel, H.R. Signer, Performance of computer-optimized tapered-roller bearings to 2.4 Million DN, J. Tribol. T ASME 103 (1981) 13-20
[15] N. Chaturbhuj, S.B. Nair, R. Tiwari, Design optimization for tapered roller bearings using genetic algorithms, In: Proceedings of the International Conference on Artificial Intelligence (IC-AI 03 2003), CSREA Press, Las Vegas, Nevada, 2003, Vol. 1, pp. 421-427,

[16] B. Walker, High speed tapered roller bearing optimization, MS Thesis, Rensselaer Polytechnic Institute, Hartford, Connecticut, 2008

[17] Z. Wang, L. Meng, H. Wensi, E. Zhang, Optimal design of parameters for four column tapered roller bearing, Appl. Mech. Mater. 63-64 (2011) 201- 204

[18] R Tiwari, K.K. Sunil, R.S. Reddy, An optimal design methodology of tapered roller bearings using genetic Algorithms, Int. J. Comp. Meth. Eng. Sci. Mech. 13 (2012) 108-127

[19] R. Tiwari, R. Chandran, Thermal based optimum design of tapered roller bearing through evolutionary Algorithm, In: Proceedings of the ASME 2013 Gas Turbine India Conference, Bangalore, Karnataka, India, 2013 (paper No. GTINDIA2013-3792).

[20] E. Dragoni, Optimal design of radial cylindrical roller bearings for maximum load-carrying capacity, Proc. IMechE, Part C: J Mech. Eng. Sci. 227 (2013) 2393-2401

[21] ISO 76, Rolling bearings - Static load ratings, 2006

[22] H. Wiesner, Rolling bearings TC4 meets GPS TC213, Evolution 19 (2012) 24-28

[23] ISO 492, Rolling bearings - Radial bearings - Tolerances, 2002

[24] K.L. Johnson, Contact Mechanics, Cambridge University Press, Cambridge, UK, 1985

[25] T.A. Harris, Rolling Bearing Analysis, 4th edn. John Wiley \& Sons, New York, 2000

[26] NSK, Bearing internal load distribution and displacement, http://www.jp.nsk.com/app01/en/ctrg/ index.cgi?gr=dn $\&$ pno=nsk_cat_e728g_5 (access date $2 / 11 / 2014)$

[27] INA, BEARINX-online Shaft Calculation, http:// www.ina.de/content.ina.de/en/products_services/ calculating/Calculation_and_testing.jsp (access date $2 / 11 / 2014$ )

[28] G. Niemann, H. Winter, B.-R. Höhn, Maschinenelemente, Springer, Vol. I Berlin, Germany, 2005

[29] ISO 355, Rolling bearings - Tapered roller bearings Boundary dimensions and series designations, 2007

[30] TIMKEN, Tapered Roller Bearing Catalogue, http:// www.timken.com/en-us/products/Pages/Catalogs . aspx (access date 24/9/2014)

[31] INA, Tapered Roller Bearing Catalogue, http://www. ina.de/content.ina.de/de/products_services/ rotativ_products/tapered_roller_bearings/ tapered_roller_bearings.jsp (access date 24/9/2014)

[32] G. Niemann, Maschinenelemente, Springer, Berlin, Germany, 1981, Vol. I 